\title{
Blockchain at the Edge: The Nexus of Capturing New Value in $5 \mathrm{G}$
}

\author{
Federico Miatton \\ Universitat Pompeu Fabra \\ Barcelona, Spain
}

\begin{abstract}
G propels mobile technology into the exclusive group of general purpose technologies that have marked the evolution of our history and triggered transformative changes in society. Edge computing is instrumental in helping $5 \mathrm{G}$ fulfil its promise and meet its expectations and requirements. This article discusses the value shifts occurred in the telecom value chain and shows how blockchain can aid value extraction in 5G, highlighting a number of services and applications of $5 \mathrm{G}$ networks that benefit from distributed ledger technology. Furthermore, the paper details a couple of applications enabled by the marriage of 5G with distributed ledger technology: autonomous unmanned (vehicular or aerial) systems and connected supply chains. The combination of 5G and edge computing permits to unlock new business value in telecom networks; in the same way that edge computing is the nexus of creating new value in 5G, blockchain is the fulcrum of capturing that new value.

Index Terms-Edge Computing, Blockchain, Distributed Ledger Technology, Telecom Value Chain, 5G, 6G, MEC, DLT, Blockchain, Business Model Innovation, Connected Supply Chain, Autonomous Unmanned Systems.
\end{abstract}

\section{INTRODUCTION}

$5 \mathrm{G}$ is poised to transform mobile into a general purpose technology [1], just like the printing press, the steam engine or electricity, which promises to impact entire industries and economies profoundly, triggering transformative changes and elevating the living standards of people around the world. $5 \mathrm{G}$ is meant to be the foundation of our digital society, providing high speed, high bandwidth and low latency connections [2], as well as connectivity to the 15 billion Internet of Things devices expected online by 2023 [3]. Compared to previous generations of communications networks, $5 \mathrm{G}$ marks a distinctive shift from merely improving the technical aspects of communications to supporting a wide array of new services and applications. Indeed, one of the main objectives of $5 \mathrm{G}$ is to open up new business opportunities in very different market sectors, called verticals, such as automotive and transportation, healthcare, media and entertainment, energy, smart cities and smart factories. One consequence of this design principle is that the $5 \mathrm{G}$ network architecture has been designed to be servicebased [4], which means in practice that all elements of its architecture have been defined through network functions that can be instantiated on demand. In a sense, $5 \mathrm{G}$ is business driven, as heterogeneous business verticals rely upon mobile connectivity for their actual implementation and deployment. This is ultimately the real novelty of $5 \mathrm{G}$ and its congenital reason to exist, and which distils the real added value brought about by 5G: its capability of creating new business opportunities and unlocking new value creation.

\section{A. Value Creation Does Not Always Imply Value Capture}

Value creation alone, however, does not imply value extraction. As a matter of fact, in the last decades, mobile data traffic has exploded, growing 1800x in the decade 2006-2016 [3] alone. To a large extent, however, mobile network operators (MNOs) have not succeeded so far to monetise this growth and the massive amount of data flowing through their networks. While operators were instrumental to the creation of value in the telecommunications ecosystem, namely by improving their 3G/4G networks, providing faster connections and larger bandwidths, and enabling new web services and apps to run on top of their networks, at the same time they failed to capture a part of the value that they contributed in creating, which resulted in stagnating or declining revenues virtually in every region [5]. At least in part, this could be attributed to the value shift that occurred throughout the same time period in the mobile sector (see Appendix A), which moved the value away from connectivity services to content, from the network infrastructure to the web services and apps running on top of it, and which have largely benefited over the-top (OTT) platform companies [6] but not network operators. 


\section{B. Yet Another Value Shift in the Telecom Value Chain}

5G brings a new exciting opportunity for MNOs, as different types of applications and traffic will be served by their network. This multiplicity of services and applications will fundamentally redefine the value framework in 5G networks and beyond, especially as machine-centric communications will be supported in 5G [7] and vertical industries start playing a bigger role in 5G. This machine-centric type of traffic that will form the backbone of the fully automated digital society of tomorrow, will represent more than half of the total number of network connections in 2022 [3], outreaching human-centric communications that have been the main focus of communications networks for the past 40 years.

The implications of this change are profound as the entire value chain in the sector is redefined (see Appendix A) and the economic opportunity grows to become huge. Estimates evaluate that the global $5 \mathrm{G}$ value chain alone will generate $\$ 3.5$ trillions in output in 2035 , and $\$ 12$ trillions of global economic activity including related industry sectors and verticals [1]. With the advent of 5G, the economic value is again shifting back to the network itself, and the services that it will enable [8]. New value creation will be driven by ubiquitous massively distributed access [9][10] on the radio access side, and by software on the core network side [11], where distributed edge clouds will play a pivotal role. It will be up to operators to make the most of this opportunity and extract the most out of this newly created value.

The rest of the paper is organized as follows: Section II gives some background on edge computing and distributed ledger technology; Section III discusses how the combination of blockchain and edge computing can be leveraged to capture new value in $5 \mathrm{G}$, highlighting the main blockchain features beneficial for mobile networks; Section IV further discusses the role of blockchain in edge computing, and their interesting interdependent and mutually beneficial relationship; Section $\mathrm{V}$ analyses two relevant business applications for 5G, and finally Section VI draws the conclusions.

\section{BACKGROUND}

In the following we provide a brief background on edge computing and distributed ledger technology.

\section{A. Multi-access Edge Computing}

The distributed cloud or multi-access edge computing (MEC) [12] aims at providing cloud computing capabilities within or close to the radio access network, which is achieved by co-locating local computing servers with base stations or access points, or at least deploying the MEC servers in their close vicinity. By shortening the physical distance between the locations where the data or the computation is consumed (the mobile device) and where it is stored or provided (the MEC server), edge computing allows for delays lower than $5 \mathrm{~ms}$ and permits to overcome the fundamental physical limitation, namely the propagation delay, that besets cloud communications and processing. Indeed, MEC is considered a key technology for $5 \mathrm{G}$ to support applications requiring at the same time extreme high data rates, low latency end-toend service provisioning, and full mobility support [13].

Edge computing permits a paradigm shift towards a more local and distributed network where services are at the centre of the system design. The edge cloud provides an efficient platform where mobile edge applications run as virtual machines on top of a virtualization infrastructure, serving new services and applications close to the mobile device and in turn enabling new business models.

\section{B. Distributed Ledger Technology}

Distributed ledger technology (DLT) or blockchain is a distributed structure that stores records of valid system changes (for example, business or user transactions) and that is replicated in a set of nodes. The nodes participating in the blockchain network connect to each other in a peer-to-peer fashion, so that no single node acts as a central point of trust or control. Hence, the blockchain enables the nodes to agree on a shared truth without the need of a central trusted entity. This shared truth is precisely the global system state that is agreed upon by all participants in the network. The blockchain has been subject of an incredible hype that no other technology has experienced hereinbefore.

Despite the huge attention it has attracted, though, the blockchain is not the result of a fundamentally new breakthrough. In fact, the building blocks the blockchain is based upon are at least ten years old, with the most fundamental ones at its core, such as cryptographic hash functions and digital signatures, being more than thirty years old. Rather, the real innovation introduced by the blockchain lays in the particular way those ingredients were cooked up together, in order to solve very specific business problems. Estonia had been experimenting and testing with this technology at least since 2007 [14]. Before the term blockchain was coined, this technology had been known in Estonia as hash-linked time-stamping and since 2008 has been in use in the country's data registries such as for the national health system and for protecting other public electronic services [15]. 


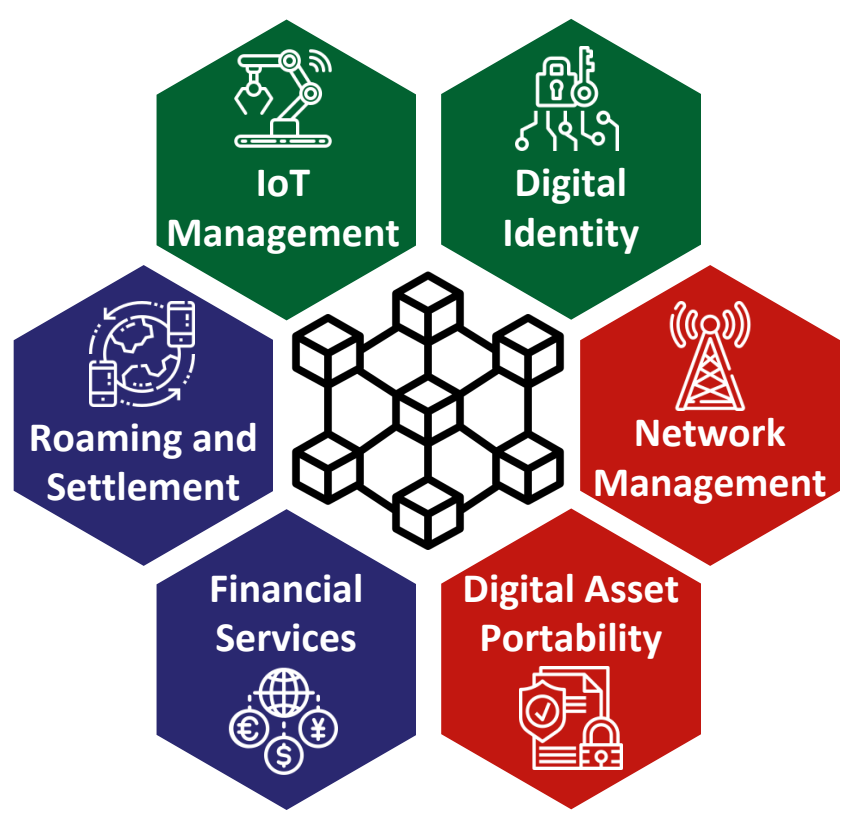

\begin{tabular}{|c|c|}
\hline $\begin{array}{l}\text { IoT } \\
\text { Management }\end{array}$ & $\begin{array}{l}\text { - Shared ledger smart cities: metered data, payment per use } \\
\text { - eSIM activation tracking and management } \\
\text { - Alerts and automated actions triggered by thresholds }\end{array}$ \\
\hline $\begin{array}{l}\text { Digital } \\
\text { ldentity }\end{array}$ & $\begin{array}{l}\text { - Decentralized identity management for individuals \& things } \\
\text { - IoT digital credentials; authentication and secure updates } \\
\text { - Identity as a Service }\end{array}$ \\
\hline $\begin{array}{l}\text { Network } \\
\text { Management }\end{array}$ & $\begin{array}{l}\text { - Automated resource and infrastructure sharing } \\
\text { - Resources provisioning, metered usage \& automated billing } \\
\text { - Enforcement \& management of Service Level Agreements } \\
\text { - NFV authentication, authorization \& automated provisioning } \\
\text { - Acquisition and planning approvals for network roll out }\end{array}$ \\
\hline $\begin{array}{l}\text { Digital Asset } \\
\text { Portability }\end{array}$ & $\begin{array}{l}\text { - Mobile number portability across mobile network operators } \\
\text { - Global data portability initiative } \\
\text { - Do not call registry }\end{array}$ \\
\hline $\begin{array}{l}\text { Financial } \\
\text { Services }\end{array}$ & $\begin{array}{l}\text { - Network for clearing \& settlement of cross-border transfers } \\
\text { - Streamlining the payments and the settlement process } \\
\text { - Dispute resolution; simplification of audit and compliance }\end{array}$ \\
\hline $\begin{array}{l}\text { Roaming \& } \\
\text { Settlement }\end{array}$ & $\begin{array}{l}\text { - Fast roaming settlements and inter-company settlements } \\
\text { - Platform for loT roaming } \\
\text { - Subscriber identification \& management, fraud prevention }\end{array}$ \\
\hline
\end{tabular}

Fig. 1: Potential applications of distributed ledger technology in 5G networks and future releases (6G).

\section{BlockChain: the NeXus of CAPturing NeW VALUE in 5G (AND 6G) NETWORKS}

Value creation is just one element of a successful business model; the other two being value delivery (not addressed here) and value capture (or extraction) [16]. On the one hand, 5G and its service-based architecture puts MNOs in a privileged position to capture the new value created through their networks. On the other hand, distributed ledger technology provides the ideal platform to do that in a seamless and autonomous fashion. Blockchain permits to automatically record events and transactions, and bundle them into blocks which are cryptographically secure, creating an immutable ledger that is shared among the different parties involved.

The advantages of implementing a DLT solution at the $5 \mathrm{G}$ edge are manifold. For instance, sharing data in a secure and transparent manner permits to reduce disputes across the different parties, and the shared ledger provided by the blockchain can be used to automate transactions and accounting. Additionally, the blockchain creates a secure record of identities not only of people but also (and especially) of things, assets, sensors and machines. Any interaction that occur with an asset can then be recorded onto the secure ledger, which in turn can be used for asset tracking, for metering or simply as a secure log. Finally, distributed ledgers offer the potential to simplify $5 \mathrm{G}$ highly complex and dynamic networks, for instance in respect to the provision of rules to access networks, and for authentication of devices onto these networks. Some applications and services that benefit from DLT systems are shown in Figure 1. Ultimately, the key features that make DLT systems attractive for mobile networks and in general for business applications are essentially three.

1) Permanency: Once information is stored on the blockchain, it cannot be deleted or modified.

2) Verifiability: All data and transactions recorded on the blockchain are verifiable, eliminating the need for a single trusted entity.

3) Transparency: The blockchain acts as an immutable ledger that is shared among all parties involved.

\section{BRINGING BLOCKCHAIN INTO EDGE Clouds}

Blockchain and MEC go hand in hand as they are both distributed by definition, and the edge cloud is the ideal place to implement DLT, given its vantage within the network, exactly where services are provided to the mobile device. For instance, a blockchain implemented at the edge can manage authentication for IoT networks, which are often fragmented into a myriad of protocols, simultaneously solving the scalability and security issues of centralised architectures. Furthermore, a DLT at the edge provides an effective platform to monetise the applications that run on the edge cloud. Applications can be billed in real time based on the precise amount of resources -communications, computing, caching [17], or a combination of these- that they use. 


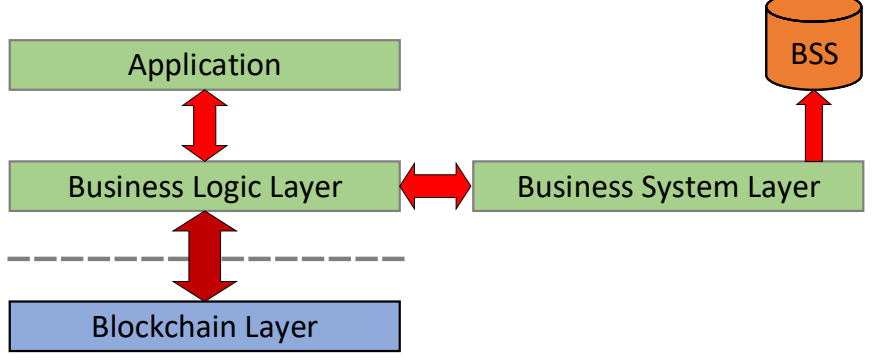

Fig. 2: High-level layered blockchain edge architecture.

A layered architecture abstracting blockchain systems in edge clouds is shown in Figure 2. The business logic layer provides a higher level interface with the blockchain network layer, providing a stratum that connects the business world with the blockchain layer and abstracting the inner parameters of the DLT in use. This layer also provides an interface for managing the $\mathrm{read} /$ write permissions of each application to interact with the ledger. For instance, an application managing the authentication of IoT sensors may only be allowed to check (read) an identity from the blockchain, whereas an asset tracking application may also be able to update (write) the current status of an asset.

The business logic layer can also calculate the precise amount of resources that an application has consumed, and communicate this information to the business system layer, which in turn generates the billing details and forwards them to the (legacy) Business Support System (BSS) of the network provider. The unit price of each resource may also be stored securely on the ledger and asked/pulled via the business logic. As blockchain platforms for communications systems become mature, the BSS itself may disappear and its functionalities integrated within the business logic layer of the DLT. Finally, the blockchain layer deals with all functionalities of the DLT at the block level, from keeping the system state consistent across peers, performing validity checks of new transactions committed to the ledger, and ensuring the immutability of past transactions.

The separation between the business logic and the blockchain layer also allows for a greater flexibility with respect to the deployment. Each layer may refer to a virtualized entity that is accessed via specified APIs, thus the blockchain network layer can run either on the edge cloud itself or somewhere else within the core network. This layered architecture also supports hierarchical implementations, where different blockchain layers run in different physical parts of the network.

\section{Automated Trust in Action: Two Relevant APPLICATIONS OF BLOCKCHAIN IN 5G}

In the prior section, we argued that the main benefits brought by distributed ledger technology revolve around its ability to provide a permanent, verifiable and transparent structure to store and share data among the network of participants. Further on, the blockchain bridges the gap in trust by automating trust-based transactions, essentially becoming a machine for creating trust in a multi-stakeholder network, being particularly useful in those where the stakeholders have trust issues or different incentives in participating in the network. Therefore, DLT effectively enables interactions between parties that do not trust each other, or when none of the parties involved wishes to be responsible for maintaining the centralised architecture needed to support such interactions. By relying on a distributed data structure that is replicated in all nodes, it implements a model of distributed trust where all nodes are responsible to ensure and verify that the interactions between peers are valid.

In the remaining of the section, we discuss how DLT can aid 5G and MEC in realising autonomous unmanned transportation systems and connected supply chains.

\section{A. Autonomous Unmanned Vehicular \& Aerial Systems}

Over the past years, vehicular road-based systems have received the most attention in the context of transportation, under an array of different names, from selfdriving cars to intelligent transport systems, autonomous vehicles, and so forth. More recently, supported by the localised processing capability offered by MEC [18] and by the cellular connectivity of 5G [19], a surge of interest in unmanned aerial vehicles (UAV) or unmanned aircraft systems (UAS) has taken place, as they have arguably the potential for the greatest impact on society. Trust is an essential feature in transportation and even more so considering fully autonomous systems; blockchain is seen as a key technology for establishing and delivering trust in this context. Although the focus of this section is on UAS, the following discussion applies to road-based and aerial systems equally.

As UAS technology gets mass adoption and UAVs become ubiquitous, air traffic control will change dramatically and a more scalable air traffic management system is needed [20]. One critical requirement of such system is the ability to register and identify drones in real-time and autonomously. Further, the ability to have a globally-accessible and transparent database will also be important. All these requirements can be met through the use of DLT for identification and authentication. 
A new concept called geofencing will also be very important in UAS, as autonomous drones will be restricted to fly in the vicinity of sensitive areas (military bases, airports, etc.) as well as in altitude. A shared secure database will also simplify the complex route management of a massive number of UAVs and will also function as a black box to keep track of liabilities.

Autonomous road-based vehicles and autonomous UAVs pave the way to new business models, such as the Mobility as a Service (MaaS) model, which combines personalised end-to-end transport services with easy digital payments, ticketing and journey planning. An electronic payment schemes based on blockchain is proposed in [21] for vehicular networks, where the payment platform is used to convert fiat currency into virtual currency to be exchanged in the automatic toll collection. A similar approach could be used for decentralised ride hailing where vehicles (or drivers until the time is ripe) could set their own fares and transact directly with customers.

\section{B. Connected Trusted and Transparent Supply Chains}

Inefficiencies in the supply chain are a pervasive problem worldwide. They negatively affect consumer pricing, the carbon footprint, food waste, and expected freshness. Often, such inefficiencies are a direct consequence of the very high cost of establishing trust in a supply chain. For instance, trading often still relies heavily on manual, cross-checked, paper-based administrative tasks to process individual trades through manual auditing, reconciliation, settlement and delivery. Another example is given by the problem of counterfeit goods: the Organisation for Economic Cooperation and Development estimates that $\$ 461$ billion worth of fake goods are sold annually, amounting to 2.5 percent of global trade [22]. In the case of food fraud, the problem is even worse as it is not only an economical one, but also an health issue. Food safety is critical and food recalls create waste, are expensive and difficult to fulfil because supply chains are opaque and not transparent at all.

A connected shared and transparent supply chain powered by blockchain enables supply chain players to better collaborate with each other and to operate more efficiently, saving costs and reducing waste. Blockchain improves accessibility and security of data, scalability, enables digital verification of goods and parties involved, and eases the regulatory validation. Increasing transparency inside the supply chain permits to work smarter across the entire shared ecosystem, eliminating bottlenecks and making possible for its participants to know the provenance of goods and create real-time demand forecasting models. This in turn permits to build more flexibility into the supply chain, which is particularly important in turbulent times such as those we live in today [23]. For instance, in [24], blockchain is used to bring transparency and traceability into the entire coffee value chain and improving its fairness as a result. Therein, DLT is used to digitise the complex and timeconsuming paper trail required to be kept as the goods move along the supply chain, as well as to deliver endto-end traceability and verified provenance throughout the entire supply chain, in order to certify the coffee's origin, quality and freshness.

\section{CONCLUSION}

5G propels mobile technology into the exclusive group of general purpose technologies that have marked the evolution of our history, and unlocks a $\$ 12$ trillion business opportunity. 5G brings yet another value shift in the mobile communications value chain, shifting once again the economic value back to the network and its software. In the same way that edge computing is the nexus of creating new value in $5 \mathrm{G}$, blockchain is the fulcrum of capturing that new value.

This article discusses the telecommunications ecosystem and its value chain, and how the value has shifted in the sector throughout the past fifty years, as Appendix A summarises. The paper describes value creation in MECenabled 5G networks, and shows how DLT can be used as a way to extract untapped value in 5G. Moreover, the paper provides an array of relevant services and applications that can be served through $5 \mathrm{G}$ networks that benefit from implementing blockchain in mobile networks. Finally, the paper describes two prominent business applications of $5 \mathrm{G}$ that make use of several features brought by distributed ledger technology.

Although throughout the paper we have mainly described blockchain as a technology to extract value, it is actually a driver for business value creation in itself. In fact, blockchain permits to save time, reduce costs, increase efficiencies and create new automated revenue streams. Blockchain can simplify the business processes and operations in complex business networks, especially when multiple parties need to share and update data that require external verification and auditing. Way too often, distributed ledger technology has been considered as a technology to resort to as a means to disrupt existing businesses; it is time to start seeing it as a technology that facilitates, optimises and automates business operations, as well as a real business value creator. 


\begin{tabular}{|c|c|c|c|c|}
\hline $1980 \mathrm{~s}$ & $1990 \mathrm{~s}$ & $2000 \mathrm{~s}$ & $2010 \mathrm{~s}$ & $2020 s$ \\
\hline 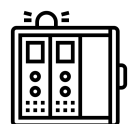 & 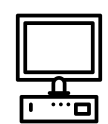 & $\stackrel{\square}{=}$ & 㗊品 & \\
\hline Mainframes & Desktops & Mobile comms & Mobile apps & Augml \\
\hline Arpanet & WWW & Telecoms & Web/Cloud & Edge cloud \\
\hline Network & Network & Network & Network & Network \\
\hline Apps & Apps & Apps & Apps & Apps \\
\hline os & os & OS & os & OS \\
\hline HW & HW & HW & HW & HW \\
\hline $\mathrm{HMI}$ & HMI & HMI & HMI & HMI \\
\hline
\end{tabular}

Fig. 3: Fifty years of value shifts in the telecommunications sector. In bold the elements that increased value in each decade; in grey those that got devalued. Figure adapted from [25] and the discussion therein.

\section{APPENDIX A}

\section{Value Shifts in the Telecom Value Chain}

Figure 3 shows the various value shifts that occurred in the telecoms ecosystem in the past fifty years [11][25].

1980s: In the era of mainframes, the elements that have value are complex hardware (HW) and the operative systems (OS) that run those supercomputers. There isn't a proper network or human machine interface (HMI) yet.

1990s: Computers move into homes and both OS and HMI matter as regular users use desktop computers for applications (apps), which are mainly office applications.

2000s: In the era of information and communication technology, mobile telephones spread. Mobile phones and networks are important; a network subscription is needed to make phone calls and OS don't matter yet.

2010s: Information and communications merge, driven by cloud systems and mobile computing, where HW, mobile OS and consumer apps are relevant. The network infrastructure gets devalued while platform [6] companies thrive, extracting most of the economic value.

2020s: In the era of augmented intelligence (AugmI) [26] and convergence of multiple technologies, HW, OS and apps disappear as everything merges into a seamless artificial intelligence (AI) system in the cloud that delivers services. Networks are paramount as $5 \mathrm{G}$ and edge clouds are essential to realise this transition. HMI too is very important as the sensors that are needed to interact with the cloud-AI become pervasive and reduce to simply bridge-APIs used to connect to the cloud.

\section{ACKNOWLEDGMENT}

This work was supported by the European Research Council under the H2020 Framework Programme/ERC grant agreement 694974, and by MINECO's Projects RTI2018-102112 and RTI2018-101040.

\section{REFERENCES}

[1] IHS Economics and IHS Technology, "The 5G economy: How 5G will contribute to the global economy," Jan. 2017.

[2] J. G. Andrews, S. Buzzi, W. Choi, S. V. Hanly, A. Lozano et al., "What will 5G be?," IEEE Journal on Selected Areas in Communications, vol. 32, no. 6, pp. 1065-1082, Jun. 2014.

[3] Cisco, "Cisco Visual Networking Index Forecast and Trends 2018-2023," Cisco White Paper, Mar. 2020.

[4] 3GPP TS 23.501, "System Architecture for the 5G System," Rel. 15, Ver. 15.4.0, Dec. 2018.

[5] B. El-Darwiche et al., Telecommunications Trends 2017, PwC Strategy, Mar. 2017.

[6] M. Van Alstyne, "Platform Shift: How New Business Models Are Changing the Shape of Industry," MIT Sloan CIO Symposium, May 2015.

[7] C. Bockelmann et al., "Towards massive connectivity support for scalable mMTC communications in 5G networks," IEEE Access, vol. 6, pp. 28969-28992, May 2018.

[8] World Economic Forum, "The Impact of 5G: Creating New Value across Industries and Society," White Paper, Jan. 2020.

[9] H. Q. Ngo et al., "Cell-free massive MIMO versus small cells," IEEE Trans. on Wireless Comm, vol. 16, no. 3, Mar. 2017.

[10] F. Boccardi, R. W Heath, A. Lozano, T. L. Marzetta, P. Popovski, "Five disruptive technology directions for 5G," IEEE Communications Mag., vol. 52, no. 2, pp. 74-80, Feb. 2014.

[11] M. Weldon, "The Future X Network: A Bell Labs Perspective," CRC Press, Mar. 2016.

[12] ETSI GS MEC 003, "Multi-Access Edge Computing (MEC); Framework and Reference Architecture," v2.1.1, Jan. 2019.

[13] V. Frascolla et al., "5G-MiEdge: Design, standardization and deployment of 5G Phase 2 technologies: MEC and mmWaves joint development for Tokyo 2020 Olympic games," 2017 IEEE Conference on Standards (CSCN), pp.54-59, Sep. 2017.

[14] E-Estonia. Available online.

[15] Guardtime Electronic Government Solutions. Available online.

[16] D. J. Teece, "Business models, business strategy and innovation,” Elsevier Long Range Planning, vol. 43, no.2-3, pp. 172194, Apr. 2010.

[17] H. Liu, Z. Chen, L. Qian, "The Three Primary Colors of Mobile Systems," IEEE Comm. Mag., vol.54, no.9, pp.15-21, Sep.2016.

[18] ETSI GS MEC 022, "Multi-Access Edge Computing (MEC); Study on MEC Support for V2X Use Cases," v2.1.1, Sep. 2018.

[19] G. Geraci et al. "Understanding UAV Cellular Communications: From Existing Networks to Massive MIMO,” IEEE Access, vol. 6, pp. 67853-67865, Nov. 2018.

[20] Distributed Sky, "Distributed Sky: Blockchain Framework for UAS Traffic Management," White Paper, Sep. 2018.

[21] X. Deng and T. Gao, "Electronic Payment Schemes Based on Blockchain in VANETs," IEEE Access, vol. 8, Feb. 2020.

[22] PwC, "Blockchain's real promise: Automating trust," MIT Technology Review Insights, Jun. 2019.

[23] E. L. Andrews and H. L. Lee, "Fasten Your Seatbelts: Turbulence Ahead for Global Supply Chains," Stanford Business Insights, Mar. 2020.

[24] F. Miatton and L. Amado, "Fairness, Transparency and Traceability in the Coffee Value Chain through Blockchain Innovation," 2020 IEEE International Conference on Technology and Entrepreneurship (ICTE), Bologna, Italy, Apr. 2020, accepted.

[25] Nokia Bell Labs Consulting and M. Weldon, "GTI Summit 2019 Keynote Speech,” GSMA MWC19 Barcelona, Feb. 2019.

[26] Y. N. Harari, "Homo Deus: A Brief History of Tomorrow," Harville Secker, 2016. 